Original Article

\title{
Activities of daily living and manual hand dexterity in persons with idiopathic parkinson disease
}

\author{
Yoo-Im Chol, $\mathrm{PhD}_{\mathrm{O}} \mathrm{OT}^{1)}$, Chiang-Soon Song, PhD, OT ${ }^{2 *}$, Byung-Yoon Chun, MBA ${ }^{3)}$ \\ 1) Department of Occupational Therapy, School of Medicine, Institute for Health Improvement, \\ Wonkwang University, Republic of Korea \\ 2) Department of Occupational Therapy, College of Health Science, Chosun University: \\ 309 Pilmun-daero, Dong-gu, Gwangju 501759, Republic of Korea \\ 3) Department of Business Administration, Graduate School, Chosun University, Republic of Korea
}

\begin{abstract}
Purpose] The purpose of this study was to evaluate the relationship between daily activities and manual dexterity in persons with Parkinson disease. [Subjects and Methods] The study participants were 25 patients with idiopathic Parkinson disease. This study used two clinical tools, the box-and-block test and Schwab and England Activities of Daily Living scale, to investigate the relationship between manual dexterity and Schwab and England Activities of Daily Living score. [Results] A positive correlation was observed between the Schwab and England Activities of Daily Living and the box-and-block test scores on the more and less affected sides. Moreover, the Schwab and England Activities of Daily Living score had a greater correlation with the box-and-block test score on the less affected side than that on the more affected side. [Conclusion] Manual dexterity and activities of daily living showed a positive correlation in individuals with Parkinson disease. The results of this study suggest that manual dexterity is an important factor for predicting physical performance in daily living in persons with Parkinson disease.

Key words: Activities of daily living, Hand, Parkinson disease
\end{abstract}

(This article was submitted Jul. 31, 2016, and was accepted Nov. 29, 2016)

\section{INTRODUCTION}

Parkinson disease (PD) is a specific chronic progressive neurodegenerative disease and is a syndrome manifested by cardinal features of resting tremor, bradykinesia, rigidity and postural instability ${ }^{1)}$. These cardinal features cause patients with PD difficulties in performing motor tasks such as hand manipulation, and increase their time to complete daily activities ${ }^{2}$. Activities of daily living (ADL) consist of daily self-care activities such as bathing and showering, dressing, self-feeding, personal hygiene, and toileting; therefore, the performance level in ADL is the most important factor to be maintained for independent living and quality of life in patients with PD. Thus, regular assessment and maintenance of performance level in ADL is crucial because PD is a progressive neurodegenerative disease and results in a decline in functional activities and independent daily living ${ }^{3)}$.

Maintenance of performance level in ADL is affected by several of motor abilities such as muscle activation, strength, power, endurance, and fatigue in the lower and upper extremities. Performance in ADL is also affected by upper and lower extremity function such as postural instability, gait disturbance, reaching and grasping, manipulation, and manual dexterity ${ }^{4}$. Therefore, these factors should be maintained for independent daily living by persons with PD. Manual dexterity is important for maintaining performance in ADL because most ADL involve the hands and some lower extremity functions substitute

*Corresponding author. Chiang-Soon Song (E-mail: grsong@chosun.ac.kr)

(C)2017 The Society of Physical Therapy Science. Published by IPEC Inc.

This is an open-access article distributed under the terms of the Creative Commons Attribution Non-Commercial No Derivatives (by-nc-nd) License $<$ http://creativecommons.org/licenses/by-nc-nd/4.0/>. 
Table 1. General and clinical characteristics of the participants $(\mathrm{N}=25)$

\begin{tabular}{lc}
\hline Variables & Participants \\
\hline Gender (male/female) & $11 / 14$ \\
Age (yrs) & $80.6 \pm 3.7$ \\
Height (cm) & $155.4 \pm 7.5$ \\
Weight (kg) & $52.0 \pm 7.4$ \\
Mini-mental state examination (scores) & $25.9 \pm 1.1$ \\
Post-disease duration (months) & $25.9 \pm 6.5$ \\
Hoehn and Yahr staging score (II/III) & $8 / 17$ \\
\hline
\end{tabular}

Table 2. BBT and SE-ADL scores of the participants $(\mathrm{N}=25)$

\begin{tabular}{lcc}
\hline Variables & Mean & $\begin{array}{c}\text { Standard } \\
\text { deviation }\end{array}$ \\
\hline BBT on the more affected side & 38.16 & 4.89 \\
BBT on the less affected side & 43.92 & 3.15 \\
SE-ADL & 74.40 & 10.83 \\
\hline
\end{tabular}

BBT: box-and-block test; SE-ADL: Schwab and England Activities of Daily Living

upper extremity functions when assistive devices such as crutches and wheelchairs are used.

Patients' daily and functional activities should be examined accurately to identify the problems they encounter in independent daily living and to plan their care at home or in clinical settings. The purpose of this study was to investigate the relationship between performance in ADL and manual dexterity in persons with PD.

\section{SUBJECTS AND METHODS}

Twenty-five persons with mild-to-moderate PD (Hoehn and Yahr staging scale score of $\leq 3$ ) participated in this study. The inclusion criteria included a diagnosis of idiopathic PD confirmed by a physician and no involvement in therapeutic trainings similar to the current intervention as judged by the occupational therapist. Patients were excluded from the study if they had a history of any other neurological or orthopedic diseases that could affect their functional activities, visuoperceptual problems that could affect the ability to use clinical measurement tools, or severe cognitive impairments that could affect their ability to follow researchers' commands. Table 1 shows the general and clinical characteristics of the participants. The participants were recruited through local rehabilitation centers and were motivated to participate in the study spontaneously. The study was conducted in accordance with the International Ethical Guidelines and Declaration of Helsinki and was approved by the local institutional review board.

This correlational research study used two clinical measurement tools, namely, the box-and-block test (BBT) and Schwab and England Activities of Daily Living Scale (SE-ADL). The BBT was developed by A. Jean Ayres and Patricia Buhler in 1957. The tool consists of a box with two square compartments of equal dimensions and 150 wooden cubes. The participant is seated at a table, facing a rectangular box, and is instructed to move as many blocks as possible, one at a time, from one compartment to the other for a period of 60 seconds. Lower scores represent lower hand function ${ }^{5)}$. The test-retest reliability of the BBT is high (intraclass correlation coefficients [ICC] of 0.89 to 0.97 ), and the validity of the BBT is shown by significant correlations between the BBT and functional independence measurement ${ }^{6)}$. The SE-ADL is a self-rated, single-item assessment of overall functional independence, describing ADL capability, and rated on an 11-point scale, from $0 \%$ (vegetative) to $100 \%$ (completely independent), in $10 \%$ increments. The duration of SE-ADL administration is 5 to 10 minutes ${ }^{7}$. The tool has been reported to have good test-retest reliability $(\mathrm{ICC}=0.7)$ and good construct validity ${ }^{8}$.

Descriptive statistics were used to analyze the data on the general and clinical characteristics of the patients, such as gender, age, height, weight, mini-mental state examination (MMSE) score, post-disease duration, and Hoehn and Yahr staging scale score. This study used the Pearson correlation to examine the relationship between BBT and SE-ADL. Multivariate logistic regression analysis was used to identify the predictors of perfoamcne in ADL in the PD patients. Analysis was performed by using PASW version 18.0 for Windows (SPSS Inc., Chicago, IL, USA), with a statistical significance level of 0.05.

\section{RESULTS}

This study examined 25 patients with idiopathic PD (11 men and 14 women; mean age, 80.64 years; mean height, $155.40 \mathrm{~cm}$; mean body weight, $51.96 \mathrm{~kg}$ ). The mean MMSE score was 25.92, and the post-disease duration was 25.92 months (Table 1). The BBT score was 38.16 on the more affected side and 43.92 on the less affected side (Table 2). The BBT on the more affected side was positively correlated with the BBT on the less affected side (0.559) and was positively correlated with SE-ADL (0.521). The BBT on the less affected side had a positive correlation with SE-ADL (0.646) (Table 3). In the multivariate regression analysis, BBT was a significant predictor of performance in ADL in the patients with idiopathic PD (Table 4). 
Table 3. Relationship between the BBT and SE-ADL scores of the participants $(\mathrm{N}=25)$

\begin{tabular}{llll}
\hline Variables & $\begin{array}{l}\text { BBT on the more } \\
\text { affected side }\end{array}$ & $\begin{array}{l}\text { BBT on the less } \\
\text { affected side }\end{array}$ & SE-ADL \\
\hline BBT on the more affected side & & $0.559^{* *}$ & $0.521^{* *}$ \\
BBT on the less affected side & $0.584^{* *}$ & & \\
SE-ADL & $0.521^{* *}$ & $0.646^{* *}$ & \\
\hline BBT: box-and-block test; SE-ADL: Schwab and England Activities of Daily Living \\
$* * \mathrm{p}<0.01$
\end{tabular}

Table 4. Multiple regression analysis between the manual dexterity and performance in activities of daily living of participants $(\mathrm{N}=25)$

\begin{tabular}{llccccccc}
\hline Variable & Independent variables & $\mathrm{B}$ & $\mathrm{SE}$ & $\beta$ & $\mathrm{t}$ & $\mathrm{R}^{2}$ & $\mathrm{~F}$ & $\mathrm{p}$ \\
\hline \multirow{2}{*}{ SE-ADL } & BBT on the more affected side & 1.775 & 0.653 & 0.516 & 2.718 & \multirow{2}{*}{0.455} & 9.178 & 0.001 \\
& BBT on the less affected side & 0.516 & 0.421 & 0.233 & 1.228 & & \\
\hline
\end{tabular}

\section{DISCUSSION}

This study investigated the correlation between performance in ADL and manual dexterity in persons with PD. The results showed a positive correlation, but performance in ADL had a greater correlation with manual dexterity on the less affected side than that on the more affected side. The result clearly shows that persons with PD tend to use their hands on the less affected side more frequently than their hands on the more affected side during daily activities.

Manual dexterity is a major factor that impairs the ability to perform daily and functional activities throughout different PD stages. Progressive impaired hand dexterity has been shown in mild to moderate PD stages typically as progressive limb bradykinesia, before the development of rigidity. This study included persons with mild to moderate PD who had scores of $\leq 3$ on the Hoehn and Yahr staging scale and had progressive impaired hand dexterity. Foki et al. attempted to identify the strongest predictor of buttoning and unbuttoning by investigating the relationship between limb-kinetic apraxia and ADL in persons with PD. The novel key finding in their study was that limb-kinetic apraxia seemed to be particularly relevant to ADL that require dexterity skills, even in mild to moderate $\mathrm{PDs}^{4}$. Mak et al. investigated the test-retest reliability of the Jebsen Taylor Hand Function Test (JTT) among older patients with PD and compared the JTT scores between patients with PD and healthy adults. They suggested that the signs and symptoms in patients with PD could lead to impairments of the upper extremities including delayed movement onset due to the reduced rate of force production, prolonged time to lift an object, and substantial impairments of hand preshaping. They reported that JTT is a reliable and easily available assessment tool for assessing the hand function of patients with PD and that patients with PD took a longer time to complete the JTT, suggesting that they had deficits in gross and fine functional dexterity ${ }^{9}$. Foster investigated the performance of cognitively demanding instrumental ADL among patients with PD who had no dementia. They reported that people with relatively early-stage and mild PD demonstrated measurable deficits in the performance of cognitively demanding instrumental ADL, highlighting the importance of using objective assessments of instrumental ADL function to detect early functional changes in people with $\mathrm{PD}^{10)}$. Previous studies have shown that both manual dexterity skills and hand function are equally important for maintaining and improving daily activities in patients with $\mathrm{PD}^{4},{ }^{10}$ ). Current study measured manual dexterity and motor function, and reported that patients with PD showed a good relationship between manual dexterity and motor function, suggesting that the more manual dexterity they had, the better their motor function. Therefore, current study suggests that the hand motor function of patients with PD can be measured by simply using the BBT instead of the clinical tool with many items to complete.

In conclusion, a positive correlation was found between manual dexterity and performance level in ADL in patients with mild-to-moderate idiopathic PD. There are some limitations in this study. First, any postural control during activities was not measured. Secondly, a disease-specific measurement tool was not used to assess the specific signs and symptoms of PD. Future study will be necessary to consider the relationship between postural stability and the coarse performance of the upper extremity as well as between symptoms (e.g., tremor, bradykinesia, and rigidity) and upper extremity function in patient with PD. Further studies are suggested to evaluate the relationship between performance in ADL and other motor functions of the upper extremity, such as reaching and grasping, manipulation, and pinch grip in persons with idiopathic PD. 


\section{REFERENCES}

1) Cholewa J, Gorzkowska A, Szepelawy M, et al.: Influence of functional movement rehabilitation on quality of life in people with Parkinson's disease. J Phys Ther Sci, 2014, 26: 1329-1331. [Medline] [CrossRef]

2) Hou JG, Lai E: Overview of Parkinson's disease: clinical features, diagnosis, and management. In: Trail M, Protas EJ, Lai EC, Neurorehabilitation in Parkinson's disease. Thorofare: SLACK Inc., 2008, pp 1-30.

3) Kim HD, Kim TY, Jae HD, et al.: The effects of Tai Chi based exercise on dynamic postural control of Parkinson's disease. J Phys Ther Sci, 2011, 23: 265-269. [CrossRef]

4) Foki T, Vanbellingen T, Lungu C, et al.: Limb-kinetic apraxia affects activities of daily living in Parkinson's disease: a multi-center study. Eur J Neurol, 2016, 23: 1301-1307. [Medline] [CrossRef]

5) Mathiowetz V, Volland G, Kashman N, et al.: Adult norms for the Box and Block Test of manual dexterity. Am J Occup Ther, 1985, 39: 386-391. [Medline] [CrossRef]

6) Desrosiers J, Bravo G, Hébert R, et al.: Validation of the Box and Block Test as a measure of dexterity of elderly people: reliability, validity, and norms studies. Arch Phys Med Rehabil, 1994, 75: 751-755. [Medline]

7) McRae C, Diem G, Vo A, et al.: Reliability of measurements of patient health status: a comparison of physician, patient, and caregiver ratings. Parkinsonism Relat Disord, 2002, 8: 187-192. [Medline] [CrossRef]

8) Dal Bello-Haas V, Klassen L, Sheppard MS, et al.: Psychometric properties of activity, self-efficacy, and quality-of-life measures in individuals with Parkinson disease. Physiother Can, 2011, 63: 47-57. [Medline] [CrossRef]

9) Mak MK, Lau ET, Tam VW, et al.: Use of Jebsen Taylor Hand Function Test in evaluating the hand dexterity in people with Parkinson's disease. J Hand Ther, 2015, 28: 389-394, quiz 395. [Medline] [CrossRef]

10) Foster ER: Instrumental activities of daily living performance among people with Parkinson's disease without dementia. Am J Occup Ther, 2014, 68: 353-362. [Medline] [CrossRef] 